\title{
SYNTHESIS AND RESEARCH OF POLYMER HYDROGELS ON THE BASIS OF HYDROLYSED POLYACRYLONITRILE AND EPICHLORHYDRIN
}

\author{
R. Zh. Omirova ${ }^{1, *}$, A. A. Bolysbek ${ }^{2}$, Sh. D. Shirinov ${ }^{3}$ and A.T. Dzhalilov ${ }^{4}$ \\ ${ }^{1}$ Department of Chemical technologies of inorganic substances of M.Auezov South Kazakhstan \\ State University, 160012, Shymkent, Kazakhstan \\ ${ }^{2}$ Department of Chemical technologies of inorganic substances of M.Auezov South Kazakhstan \\ State University, 160012, Shymkent, Kazakhstan \\ ${ }^{3}$ Tashkent Scientific Research Institute of Chemical Technology, Tashkent, Uzbekistan \\ ${ }^{4}$ Tashkent Scientific Research Institute of Chemical Technology, Tashkent, Uzbekistan \\ *E-mail:nauka3524@gmail.com
}

\begin{abstract}
The article studies the synthesis of a highly swelling polymer hydrogel based on the interaction of epichlorohydrin with HYPAN. There was analyzed the influence of temperature and the concentration of initial reagents on gelforming in the reaction mixture and swelling degree of the extracted hydrogel, also were established optimal conditions for hydrogel synthesis. The swelling kinetics of a hydrogel-based on HYPAN (hydrolyzed polyacrylonitrile) and epichlorohydrin were learned, and there was shown the optimum degree of grinding during the process of the hydrogel swelling in water.

The article explains information on the synthesis of new macromolecular hydrogel based on HYPAN(hydrolyzed polyacrylonitrile) and CMTH (Carboxymethylcellulose) with new sewing agents, looked through synthesized products and determined their structure by various Physico-chemical and physic mechanical methods, shows the making and developing of industrial production of import-substituting products from local raw materials and industrial wastes to saturate the domestic market. The resulted hydrogel is used where there is not enough water, in places where the earth is rich in salts, to save mineral fertilizer and other spheres for the complete or partial solution of the problems.
\end{abstract}

Keywords: HYPAN (hydrolyzed polyacrylonitrile), Polymers, Temperature, Epichlorohydrin, Hydrophilic

(C) RASĀYAN. All rights reserved

\section{INTRODUCTION}

The geographical position of the Republic of Uzbekistan determines its natural peculiarity; irrigative agriculture is the main source of food production, which accounts for $85.0 \%$ of the current volume of water in the republic. Consequently, raises the question of rational use of flowing storage. It becomes aware greater because of the Aral problem and the growth of inhabitants. The above-pointed facts compelrural economy to turn to water-saving technologies in the future. One way of expedient use of flowing storage is the use of highly swelling polymer hydrogels that is of current importance from an ecological and economic standpoint.

Such highly swelling polymer hydrogels extracted on the basis of a polyelectrolyte complex of polyacrylic acid-polyethylene amine ${ }^{1}$, vinyl esters of glycols $^{2}$ with polyacrylonitrile $(\mathrm{PAN})^{3}$ polyacrylamide (PAA) ${ }^{4}$ and hydrolyzed polyacrylonitrile (HYPAN). ${ }^{5}$

While epichlorohydrin`s interaction with HYPAN (hydrolyzed polyacrylonitrile), as well as with ammonia, both water-soluble oligomers, polymers and non-water-soluble polymers of the cross-linked structure are extracted with valuable physicochemical, physicomechanical properties which are widely used in various industries (chemical, textile, metallurgical, Pharmaceutical), and in agriculture. ${ }^{6-9}$ As a

Rasayan J. Chem., 12(4), 2047-2051(2019)

http://dx.doi.org/10.31788/RJC.2019.1245331

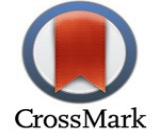


result of this, the study of these products causes some positive scientific and practical interest. These polymers are extracted by means of modification HYPAN`s hydrophilic molecules with various crosslinking agents. ${ }^{10-11}$ The macromolecules of these polymers contain polar units, therefore, the swelling of this hydrogel depends on the ionic strength of the solution.

\section{EXPERIMENTAL}

HYPAN`s (hydrolysis of polyacrylonitrile) synthesis was conducted as follows: $110 \mathrm{ml}(0.22 \mathrm{~mol})$ of $8 \%$ sodium hydroxide solution were poured in a round-bottomed $300 \mathrm{ml}$ flask equipped with a reflux condenser, then was filled up with $10 \mathrm{~g}(0.2 \mathrm{~mol})$ of polyacrylonitrile reduced to fine particles. The mixture was heated in an oil bath till coming to a boil (bath temperature 100-110 ${ }^{\circ} \mathrm{C}$ ) and held it for about 13 hours. The heating was stopped when the solution became colorless and clear. The solution was cooled and poured into a $500 \mathrm{ml}$ beaker, neutralized $0.5 \mathrm{~N}$ with hydrochloric acid, $\mathrm{pH} 8$ to 2 on the universal indicator.

The sodium polyacrylate was precipitated from the solution by the addition of methanol in volume $150 \mathrm{ml}$. The resulting precipitate was filtered on a Buchner funnel and washed with methanol until the traces of chlorides in the mother liquor disappeared. The purified sodium polyacrylate was dried in air for 48 hours. About $15.6 \mathrm{~g}$ of the polymer was the output. Sodium polyacrylate is a white soluble in water powder.

By acidification of a 5\% aqueous solution of sodium polyacrylate with an equivalent amount of $10 \%$ hydrochloric acid was extracted free polyacrylic acid. The precipitated polyacrylic acid was washed with water until the traces of chlorides in the mother liquor disappeared and then dried in air for 48 hours. Synthesis of hydrogel based on HYPAN and epichlorohydrin. $10 \mathrm{ml}$ of HYPAN was placed in a flask equipped with a mixer, a thermometer and a dropping funnel, and, keeping up the temperature of about $15-20{ }^{\circ} \mathrm{C}$ while intense stirring was slowly added dropwise $0.1 \pm 3.0 \%$ of the cross-linking agent, epichlorohydrin. Then the solution was stirred for several hours at room temperature, after which the formed hydrogel was dried in vacuum at the same temperature.

\section{RESULTS AND DISCUSSION}

The synthesis of ionic polymers is influenced by various factors, and in order to study these regularities, we investigated the effect of concentration, the ratio of the source products and the duration of the polymerization reaction of GIPAN with epichlorohydrin.

As the solubility of epichlorohydrin in various solvents is different, we chose as a solvent system the water, which supported the homogeneity of the solution.

While correlating of components polymers with the high exit of polymers are extracted, where even if one of them is in surplus, the polymer will be synthesized with an equal ratio of components in the finished product. The temperature variations were chosen by the selection, in order to reduce to minimal losses and the consumption of reagents for side reactions. Certain regularity delineates when epichlorohydrin reacts with HYPAN, which makes it possible to characterize the effect of temperature on the polymer yield, i.e. the increase in temperature increases the yield of the polymer, with a change in the concentration of the starting reagents at room temperature, after a certain period of time, the reaction temperature gradually raises.

At very high solution concentrations and high temperatures, the reaction happens almost momentary and proceeds at a high rate, resulting in a strongly crosslinked polymer at very high solution concentrations and high temperatures. The analyzing of spontaneous polymerization, which takes place during the interaction of ECG with HYPAN, shows that the same picture is observed with increasing temperature and the degree of reaction of the components proceeds almost instantaneously. It should be noted that in all studying systems the reaction occurs exothermically.

Investigation of the HYPAN`s crosslinking was carried out in aqueous solutions, in the presence of a potassium persulfate initiator. The amount of the cross-linking agent was varied between $0.1-5.0 \%$ by weight of HYPAN. At temperatures above $60^{\circ} \mathrm{C}$, the reaction proceeds in a short time as the change in the reaction temperature have a noticeable effect on the entire gelation process, so. Numerous experiments have chosen the optimum reaction temperature for HYPAN with epichlorohydrin $80^{\circ} \mathrm{C}$, at 
RASĀYAN J. Chem.

Vol. 12 | No. 4 |2047 - 2051| October - December | 2019

which gelling occurs in a few minutes and swells the resulting hydrogel higher than the hydrogel extracted at a different temperature (Table-1).

Table -1: Influence of the Reaction Temperature of the Preparation of Hydrogel HYPAN: ECG on the Degree of Swelling

\begin{tabular}{|c|c|c|c|c|c|c|}
\hline \multirow{3}{*}{ № } & \multirow{3}{*}{ HYPAN, $\%$} & \multirow{3}{*}{ ECG, $\%$} & \multicolumn{4}{|c|}{ Reaction Temperature } \\
\hline & & & $40^{\circ} \mathrm{C}$ & $60^{\circ} \mathrm{C}$ & $80^{\circ} \mathrm{C}$ & $100^{\circ} \mathrm{C}$ \\
\hline & & & \multicolumn{4}{|c|}{ Degree of Swelling } \\
\hline 1 & 99,0 & 1,0 & 70 & 120 & 360 & 320 \\
\hline 2 & 98,0 & 2,0 & 150 & 215 & 420 & 380 \\
\hline 3 & 97,0 & 3,0 & 170 & 370 & 500 & 450 \\
\hline 4 & 96,0 & 4,0 & 200 & 250 & 370 & 320 \\
\hline 5 & 95,0 & 5,0 & 245 & 240 & 300 & 260 \\
\hline
\end{tabular}

As indicated in the table the degree of hydrogels swelling depends on the reaction temperature; at the temperature of $80^{\circ} \mathrm{C}$ and in a ratio of HYPAN: ECG 97.0\%: 3.0\%, the degree of swelling is 500 .

The effect of the change in the concentration of the cross-linking agent on the swelling of the polymer was examined. Experiments have proved that while increasing the concentration of the cross-linking agent, the degree of hydrogel`s swelling is maximized, this is because there will be formed a dense net of cross-linked sections when the concentration of the cross-linking agent is increased, which in turn reduces the water-absorbing capacity of the synthesized hydrogels.

Table-2: The Effect of the Crosslinking Agent Concentration on the Swelling of the Polymer

\begin{tabular}{c|c|c|c|c|c}
\hline № & GIPAN, \% & ECG,\% & $\begin{array}{c}\text { Degree of } \\
\text { Swelling }\end{array}$ & $\begin{array}{c}\text { Ratio of } \\
\text { Communications and } \\
\text { Links }\end{array}$ & $\begin{array}{c}\text { Communication } \\
\text { /link, } \mathrm{n}\end{array}$ \\
\hline 1 & 99,9 & 0,1 & 240 & $1: 999$ & 0,001 \\
\hline 2 & 99,7 & 0,3 & 250 & $1: 333$ & 0,003 \\
\hline 3 & 99,5 & 0,5 & 270 & $1: 198$ & 0,005 \\
\hline 4 & 99,3 & 0,7 & 290 & $1: 141$ & 0,007 \\
\hline 5 & 99,1 & 0,9 & 320 & $1: 111$ & 0,009 \\
\hline 6 & 99,0 & 1,0 & 360 & $1: 99$ & 0,01 \\
\hline 7 & 98,0 & 2,0 & 420 & $1: 51$ & 0,019 \\
\hline 9 & 97,0 & 3,0 & 500 & $1: 33$ & 0,03 \\
\hline 10 & 96,0 & 4,0 & 370 & $1: 24$ & 0,04 \\
\hline
\end{tabular}

The influence of the hydrogel synthesis temperature on the degree of swelling was also studied (Table-2). At low concentrations of the cross-linking agent, the dependence has a directly proportional character, while an increase in a concentration above 3.0\%, infringes this dependence. The concentration of the cross-linking agent in the reaction mixture within the limits of 2.0-4.0\% is optimal, as with its increasing, the degree of macromolecules cross-linking of the formed polymer increases and, consequently, the degree of hydrogel's swelling reduces.

There was examined the influence of the initial reagents' concentration on gelling in the reaction mixture. With increasing of the dilution's degree of the reaction mixture, being observed either the absence of a gelling process or the process is strongly delayed in time. In this case, the product has a very low degree of cross-linking and is generally soluble in water.

Experimentally was chose the optimum concentration of HYPAN in the solution to $11.0-20.0 \%$. Magnification in a concentration above $20.0 \%$ leads to an increase in the viscosity of the solution, which complicates the process, on the one hand, mixing, and, consequently, a decrease in the reactivity of the mixture as a whole.

As well the most significant properties of the extracted hydrogel were determined for the perfect characterization of these polymers. Since it is planned to operate hydrogels for several years, it has 
RASĀYAN J. Chem.

Vol. 12 | No. 4 |2047 - 2051| October - December | 2019

become necessary to study the multiplicity of swelling. It is obvious that hydrogel's water-absorbing capacity decreases slightly when the aggregate state of the hydrogel is changed from the maximum swelling to the minimum. So, one of the criteria for evaluating the hydrogel for its application is the multiplicity of swelling. We conducted experiments to examine the hydrogel samples' behavior experienced repeated swelling and drying at room temperature.

The degree of breakage of the hydrogel strongly affects the process of hydrogel`s swelling (Table-3).

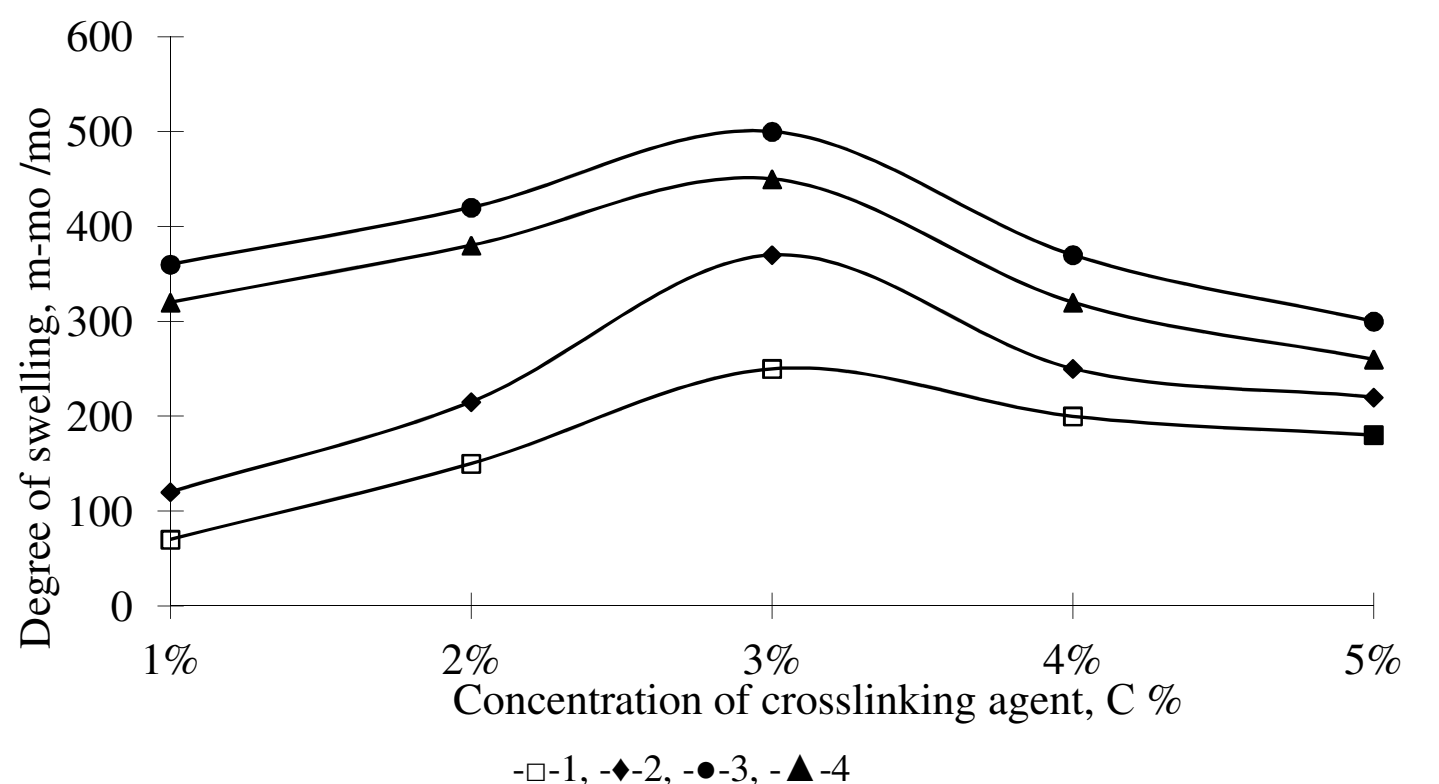

Fig.-1: Influence of the Cross-Linking Agent On The Degree of Swelling of the Synthesized Hydrogel Based on HYPAN: ECG at Different Temperatures, ${ }^{0} \mathrm{C}: 1-40 ; 2-60 ; 3-80 ; 4-100$. No. 1

As the Table- 3 shows, when the hydrogel is crushed to $0.2 \mathrm{~mm}$, the degree of its swelling reaches 350 in 30 minutes, the hydrogel with an average grinding fraction from $0.2 \mathrm{~mm}$ to $1.0 \mathrm{~mm}$ swells to 350 in 50 minutes, and particles more than $1,0 \mathrm{~mm}$, the swelling degree 320 is reached in only 60 minutes. It can be concluded that in the fine fraction of the cross-linking agent, the swelling time reaches a maximum twice as fast as particles with granules of more than $1.0 \mathrm{~mm}$ (Fig.-2).

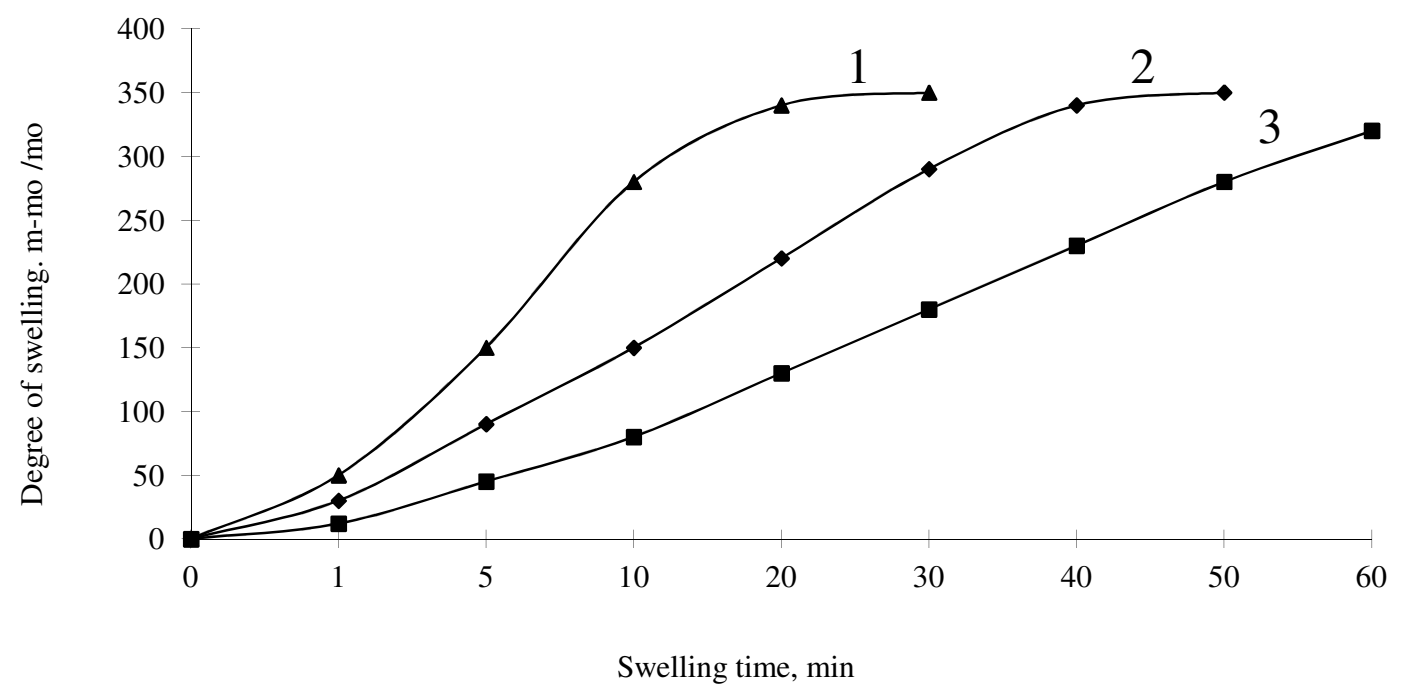

Fig.-2: Dependence of Granulation Degree on the Swelling Kinetics of the Hydrogel-based on HYPAN and Epichlorohydrin: 1 - Fine Fraction up to $0.2 \mathrm{~mm} ; 2$ - Middle Fraction, from $0.2 \mathrm{~mm}$. Up to 1.0; 3 - Coarse Fraction, Granular Form - more than 1.0 mm. No. 2 
RASĀYAN J. Chem.

Vol. 12 | No. 4 | 2047 - 2051| October - December | 2019

Table -3. Kinetics of Swelling of a Hydrogel-based on HYPAN and Epichlorohydrin

\begin{tabular}{c|c|c|c|c|c|c|c|c|c}
\hline № & Degreeofgrinding & \multicolumn{7}{|c}{ Swelling time, min. (Distilled water $20^{\circ} \mathrm{C}$. } \\
\cline { 3 - 21 } & 1 & 5 & 10 & 20 & 30 & 40 & 50 & 60 \\
\hline 1 & Fine fraction up to $0.2 \mathrm{~mm}$. & 50 & 150 & 280 & 340 & 350 & & & \\
\hline 2 & $\begin{array}{c}\text { Average fraction, from } 0.2 \mathrm{~mm} \\
\text { up to } 1.0 \mathrm{~mm}\end{array}$ & 30 & 90 & 150 & 220 & 290 & 340 & 350 & \\
\hline 3 & $\begin{array}{c}\text { A large fraction, granular - } \\
\text { more than } 1.0 \mathrm{~mm} .\end{array}$ & 12 & 45 & 80 & 130 & 180 & 230 & 280 & 320 \\
\hline
\end{tabular}

\section{CONCLUSION}

Thus, we synthesized water-swelling and water-insoluble polymers based on HYPAN. In the synthesis as a result of the formation of the structure of grid polymers, the main method is cross-linking, since it determines the mesh size of the formed hydrogel and regulates the length of chain, having a decisive influence on the properties of the polymer.

\section{REFERENCES}

1. M. Kopylova Ye., S.P. Valuyeva, B.S. El'tsefon, V.B. Rogacheva, Zezin High-altitude A.B..Comm., Moscow, 3, 517(1987)

2. G.A.Mun, Z.S.Nurkeyeva, B.B.Yerukhambetova, R.A.Abdykalykova, Ye.M. Shaykhutdinov KazNU Bulletin, Alma Ata, 12(4), 58(1998), DOI: 10.1016/S0969-806X(01)00445-5

3. A. Pourjavadi, H. Hosseinzaden, A. M. Harzandi, Polymer Congress MACRO 2004, World 40 ${ }^{\text {th }}$ International Symposium on Macromolecules, Paris p.802. (2004.)

4. I. K.Grigor'yants, G.A. Trikhanova Moskva: Znaniye, 64 (3), 204 (1984.)

5. Zhenbin Chen, Mingzhu Liu, Songmei Ma. Reactive \& Functional Polymers., 62 (1), 85(2005), DOI: $10.1016 /$ j.reactfunctpolym.2004.09.003

6. I. Ohmine, T. Tanaka, Journal Chem. Phys., 77 (11), 5725(1982), DOI: 10.1063/1.443780

7. Liu. Xinxing, Tong. Zhen, $\mathrm{Hu}$. Ou, Macromolecules, 28(11), 3813(1995), DOI: 10.1021/ma00115a010

8. M. Casolaro, Polymer Congress MACRO 2004. World 40th International Symposium on Macromolecules. July 4-9, 2004, Paris 3231 (2004)

9. N.A. Peppas, Current Applications and Future Trends, 18 (5), 41(1993)

10. I. Gustian, Ghufira and D. Oktiarni, Rasayan J. Chem., 12(1), 284(2019), DOI:10.31788/RJC.2019.1215010

11. V.M. Shevko, Badikova, D.D. Amanov, G.E. Karataeva, B.A. Lavrov, Rasayan J. Chem., 11 (3), 1050(2018), DOI: 10.31788/RJC.2018.1132038

[RJC-5331/2019] 